\title{
CT-based radiomics scores predict response to neoadjuvant chemotherapy and survival in patients with gastric cancer
}

\section{Kai-Yu Sun}

The First Affiliated Hospital of Sun Yat-sen University

Hang-Tong $\mathrm{Hu}$

The First Affiliated Hospital of Sun Yat-sen University

\section{Shu-Ling Chen}

The First Affiliated Hospital of Sun Yat-sen University

Jin-Ning Ye

The First Affiliated Hospital of Sun Yat-sen University

\section{Guang-Hua Li}

The First Affiliated Hospital of Sun Yat-sen University

\section{Li-Da Chen}

The First Affiliated Hospital of Sun Yat-sen University

\section{Jian-Jun Peng}

The First Affiliated Hoapital of Sun Yat-sen University

\section{Shi-Ting Feng}

The First Affiliated Hospital of Sun Yat-sen University

\section{Yu-Jie Yuan}

The First Affiliated Hospital of Sun Yat-sen University

\section{Xun Hou}

The First Affiliated Hoapital of Sun Yat-sen University

Hui Wu

The First Affiliated Hospital of Sun Yat-sen University

\section{Xin Li}

The First Affiliated Hospital of Sun Yat-sen University

\section{Ting-Fan Wu}

The First Affiliated Hospital of Sun Yat-sen University

\section{Wei Wang ( $\nabla$ wangw73@mail.sysu.edu.cn )}

Department of medical ultrasonics, the first affiliated hospital of Sun Yat-Sen University https://orcid.org/0000-0003-0685-2311

\section{Jian-Bo Xu}

The First Affiliated Hospital of Sun Yat-sen University 
Research article

Keywords: Stomach Neoplasms; Neoadjuvant Therapy; Tomography, X-Ray Computed

Posted Date: May 19th, 2020

DOI: https://doi.org/10.21203/rs.2.17517/v3

License: (c) (i) This work is licensed under a Creative Commons Attribution 4.0 International License. Read Full License

Version of Record: A version of this preprint was published at BMC Cancer on May 25th, 2020. See the published version at https://doi.org/10.1186/s12885-020-06970-7. 


\section{Abstract}

Background: Neoadjuvant chemotherapy is a promising treatment option for potential resectable gastric cancer, but patients' responses vary. We aimed to develop and validate a radiomics score (rad_score) to predict treatment response to neoadjuvant chemotherapy and to investigate its efficacy in survival stratification.

Methods: A total of 106 patients with neoadjuvant chemotherapy before gastrectomy were included (training cohort: $n=74$; validation cohort: $n=32$ ). Radiomics features were extracted from the pre-treatment portal venous-phase CT. After feature reduction, a rad_score was established by Randomised Tree algorithm. A rad_clinical_score was constructed by integrating the rad_score with clinical variables, so was a clinical score by clinical variables only. The three scores were validated regarding their discrimination and clinical usefulness. The patients were stratified into two groups according to the score thresholds (updated with post-operative clinical variables), and their survivals were compared.

Results: In the validation cohort, the rad_score demonstrated a good predicting performance in treatment response to the neoadjuvant chemotherapy $(\mathrm{AUC}[95 \% \mathrm{Cl}]=0.82[0.67,0.98])$, which was better than the clinical score (based on pre-operative clinical variables) without significant difference $(0.62[0.42,0.83]$, $P=0.09)$. The rad_clinical_score could not further improve the performance of the rad_score $(0.70[0.51$, 0.88], $P=0.16)$. Based on the thresholds of these scores, the high-score groups all achieved better survivals than the low-score groups in the whole cohort (all $P<0.001$ ).

Conclusion: The rad_score that we developed was effective in predicting treatment response to neoadjuvant chemotherapy and in stratifying patients with gastric cancer into different survival groups. Our proposed strategy is useful for individualised treatment planning.

\section{Background}

Gastric cancer remains the third most frequent cause of cancer-related death worldwide, resulting in 782,685 deaths annually [1]. Despite the improvement in screening, a large proportion of patients in China are diagnosed at advanced stage. For locally advanced cases, the 5-year survival rate ranged from $20 \%$ to $30 \%$ after curative resection [2-4].

Given this poor prognosis, neoadjuvant chemotherapy has been tried for this patient population in recent years. After the promising results obtained with "MAGIC Trial", "FFCD Trial", "ACCORD Trial", and "AIOFLOT3 Trial", neoadjuvant chemotherapy has become a promising treatment option for potentially resectable or limited metastatic gastric cancer with the improved 5-year survival rates of more than $35 \%$ [5-10]. Despite the satisfactory efficacy of neoadjuvant chemotherapy, patients' responses varied between $30 \%$ and $60 \%$ [11]. A good response to neoadjuvant chemotherapy was associated with good survival outcome, while non-responding patients could suffer from adverse events and unnecessary costs and finally risk tumour progression and even miss the chance to undergo curative gastrectomy. Moreover, patients who are non-responsive to neoadjuvant chemotherapy could be waiting longer until surgery, and 
this extended time to surgery may be correlated with poorer survival of gastric cancer. Thus, early detection of those patients who are most likely to respond to neoadjuvant treatment is critical to provide them a chance for a timely surgery and to optimise the treatment plans. However, the treatment efficacy

of neoadjuvant chemotherapy can only be assessed after three cycles of treatment. Therefore, exploring the pre-treatment predictors of treatment efficacy is important to determine the need for neoadjuvant therapy and the optimal timing for surgical resection, thus improving pre-treatment decision making.

Previous studies have investigated several imaging modalities such as contrast enhanced ultrasound, computed tomography (CT), magnetic resonance imaging, and positron emission tomography in the evaluation of patients' response to chemotherapy for gastric cancer; however conflicting results were obtained [12-18]. Additionally, in these studies, analyses were only based on imaging features extracted by naked eyes or quantitative imaging parameters, and lacked a proper validation. Although naked eyes provide valuable feature information, some microcosmic imaging features relevant for clinical outcomes might be lost due to the limited visual image grey scales that can be detected by naked eyes. Radiomics is a rapidly growing discipline based on high-throughput quantitative image analysis to characterise tumours and their microenvironment. This approach can extract far more features than manual extraction by acquiring two-dimensional and high-dimensional imaging features using computer algorithm [19]. Many studies on other cancer types showed that radiomics features, such as texture features, filter transformed features, wavelet features, and so on, could not be visually observed but were closely related to pathologic microscopic structures and were effective in prognostic prediction [20-23].

Computed tomography is the preferred imaging examination for gastric cancer in clinical practice, but no literature has been reported on the application of CT-based radiomics technique to predict the response to neoadjuvant chemotherapy in gastric cancer patients. Therefore, we aimed to develop and validate a CTbased radiomics score to predict the response to neoadjuvant chemotherapy and stratify the survival for patients with gastric cancer.

\section{Methods}

\section{Patients}

Consecutive patients diagnosed with gastric cancer between January 2010 and December 2017 were identified by reviewing the database of the Center of Gastrointestinal Surgery of the First Affiliated Hospital of Sun Yat-Sen University. Patients were included according to the following criteria: (1) histologically confirmed gastric adenocarcinoma on gastroscopy; (2) potential resectable gastric cancer at clinical stage of III, IV as determined by pretreatment contrast-enhanced CT (patients with M1 were those with only para-aortic lymph node metastasis without any other risk of curative resection); (3) received neoadjuvant chemotherapy of SOX regimen (S-1 plus oxaliplatin) as the initial treatment; (4) underwent curative gastrectomy; (5) received contrast-enhanced CT within one week before neoadjuvant chemotherapy; (6) Eastern Cooperative Oncology Group performance status between 0 to 1 ; (7) a life expectancy of $>3$ months; (8) adequate bone marrow, renal, and hepatic function [platelets $>80 \times 10^{9} / \mathrm{L}$, 
absolute neutrophil count $\geq 1.5 \times 10^{9} / \mathrm{L}$, serum creatinine $\leq 1.5 \mathrm{mg} / \mathrm{dL}$, total bilirubin level within $1.5 \times$ the upper limit of normal (ULN), and serum transaminase $\leq 2.5 \times \mathrm{ULN}]$. The following exclusion criteria were used: (1) history or presence of other malignancies; (2) presence of other uncontrolled diseases or severe infection; (3) received other anti-tumour therapies before neoadjuvant chemotherapy; (4) incomplete clinical data. The patient selection process is shown in Figure 1. Patients were randomly allocated to the training and validation cohorts at the ratio of 7:3. Our Institutional Ethic Review Board has approved the current study, following the regulations outlined in the Declaration of Helsinki.

\section{Neoadjuvant chemotherapy}

Patients received the first-line neoadjuvant chemotherapy of SOX regimen. S-1 was orally administered twice daily at concentrations based on body surface area (BSA): BSA $<1.25 \mathrm{~m}^{2}, 80 \mathrm{mg} / \mathrm{d} ; 1.25 \mathrm{~m}^{2} \mathrm{BSA}<$ $1.50 \mathrm{~m}^{2}, 100 \mathrm{mg} / \mathrm{d}$; and $B S A \geq 1.50,120 \mathrm{mg} / \mathrm{d}$. On the first day, oxaliplatin $\left(130 \mathrm{mg} / \mathrm{m}^{2}\right)$ was administered via intravenous infusion, followed by $\mathrm{S}-1$ administered for 14 consecutive days, followed by a 1-week break for a maximum of three cycles, until tumour progression, presence of unacceptable toxicity or treatment withdrawal by the patient or doctor.

\section{Assessment of the response to neoadjuvant chemotherapy}

The treatment response to neoadjuvant chemotherapy was evaluated via pathologic response. Haematoxylin and eosin-stained slides were reviewed by two pathologists with more than 10 years of experience in gastrointestinal pathology who were blinded to the clinical data, and they graded the specimens for pathologic response according to the Mandard tumour regression grading (TRG) system [24]. TRG 1 was defined as complete regression/fibrosis with no viable tumour cells, TRG 2 was defined as fibrosis with scattered tumour cells, TRG 3 was defined as fibrosis and tumour cells with predominant fibrosis, TRG 4 was defined as fibrosis and tumour cells with predominant tumour cells, and TRG 5 was defined as tumour without evidence of regression. Disagreement was resolved by discussion with consensus. Responders were defined as TRG 1-2 and non-responders were defined as TRG 3-5 [25].

\section{CT images acquisition}

The standard dynamic contrast-enhanced MDCT scan (Aquilion 64; Toshiba Medical System, Tokyo, Japan) procedure was used. Briefly, after an unenhanced helical sequence scan from the liver dome to the symphysis pubis, venous phase contrast-enhanced CT was performed after a 65 -s delay following intravenous administration of $80-100 \mathrm{ml}(1.5 \mathrm{ml} / \mathrm{kg})$ of iodinated contrast agent (Ultravist 300; Schering, Berlin, Germany) administered via the antecubital vein at a rate of $2.0-3.0 \mathrm{ml} / \mathrm{s}$. The following CT acquisition parameters were used: $120 \mathrm{kV}, 200-250 \mathrm{mAs}$, rotation time of $0.5 \mathrm{~s}$, collimation of $64 \mathrm{~mm} \times$ $0.5 \mathrm{~mm}$, slice thickness of $0.5 \mathrm{~mm}$, slice increments of $0.5 \mathrm{~mm}$, pitch of 0.9 , field of view of $350 \times 350$ $\mathrm{mm}$, matrix of $512 \times 512$, and reconstruction thickness of $2.5 \mathrm{~mm}$. CT images were retrieved from the picture archiving and communication system (PACS) (HP workstation XW8200, VitreaCore, version 3.7) for image analysis. The display window width was $150-350 \mathrm{HU}$, and the window level was 50 to $80 \mathrm{HU}$. 
One such case is presented in Figure 2 with CT images before and after the neoadjuvant chemotherapy and the image of response assessment by pathology.

\section{Radiomics feature extraction}

Portal venous phase contrast-enhanced CT images were used for radiomics feature extraction because of the better differentiation between the tumour tissue and the adjacent normal tissue of the gastric wall in the portal venous phase than in arterial phase. A region of interest (ROI) was delineated around the tumour outline for the largest cross-sectional area while excluding the air area by two independent radiologists with more than five years of experience in gastrointestinal imaging, and any disagreements were resolved by the consensus with arbitration by a third author. For each ROI, a total of 1044 imaging features were extracted and analyzed by an in house-made software: the A.K. software (Analysis-Kit, version 2.0.0, GE healthcare), which included six kinds of features (Supplemental Table 1): 42 histogram parameters, 10 texture parameters, 9 form factor parameters, 432 grey level co-occurrence matrix (GLCM), 540 grey level run-length matrix (GLRLM), and 11 grey level Size Zone Matrix (GLSZM).

\section{Feature reduction and model building}

The included patients were divided into the training and validation cohorts by a ratio of 7:3 using randomstratified grouping. In the training cohort, support vector machine (SVM) and principle component analysis (PCA) were used to select significant radiomics features in the tumour associated with patient response to neoadjuvant chemotherapy $[26,27]$. Based on the selected radiomics features, the Extremely Randomised Tree (Extra-Trees) method was applied to construct the radiomics score (rad_score) [28,29]. The detailed Extra-Trees method is described in the Supplemental Materials. Then, the clinical variables were selected for the univariable and multivariable logistic regression models based on the backward selection with $P$-values less than 0.05 in the training cohort. A clinical score was formulated based on the clinical variables selected from the multivariable model. The significant clinical variables and radiomics score were integrated to establish the rad_clinical_score.

\section{Model evaluation and comparison}

All the three scores were applied to classify responders and non-responders to neoadjuvant chemotherapy, and the results were validated in the validation cohort. The diagnostic ability of these scores was assessed with the area under the characteristics operating curves (AUC), accuracy, sensitivity, specificity, positive predictive value, and negative predictive value. The comparisons of these scores in predicting responders to neoadjuvant chemotherapy were performed using the AUCs and decision curve analysis (DCA). DCA was conducted to determine the clinical usefulness of these scores by quantifying the net benefits at different threshold probabilities.

\section{Survival analysis}

In the whole cohort, the clinical score and rad_clinical_score were updated with post-operative clinical variables. Univariable and multivariable Cox regression analyses were performed to investigate the 
prognostic effects of rad_score, updated clinical score, and rad_clinical_score. According to the thresholds obtained when the Youden index was the largest, patients were stratified into high-score and low-score groups respectively by the above three scores. Kaplan-Meier curves were plotted and survival rates were compared between two groups using log-rank tests.

\section{Statistical analyses}

The feature reduction and model building were performed in Python (version 2.7.14), utilising ExtraTreesClassifier from Scikit-learn. Other statistical analyses were performed by R software version

3.2.3 (R Foundation for Statistical Computing, Vienna, Austria, https://www.R-project.org/). The continuous variables were presented as mean \pm standard deviation or median and quartile, and the categorical variables were presented as frequencies and percentage. Independent sample t-test or Kruskal-Wallis (KW) nonparametric rank sum test was used to compare the baseline characteristics between the training and validation cohorts, and between responding group and non-responding group for continuous variables, while Chi-square test or Fisher exact test for categorical variables. A two-sided $P$-value was considered statistically significant if less than 0.05 .

\section{Results}

\section{Baseline characteristics}

A total of 106 patients were included, with 74 patients in the training cohort and 32 in the validation cohort. These two cohorts were comparable in baseline characteristics (Table 1). The median time interval between the surgery and chemotherapy was 73 days (range, 70-77 days) in the training cohort and 74 days (range, 70-77) in the validation cohort.

\section{Model construction}

In the training cohort, SVM and PCA analysis identified 25 radiomics features significantly associated with the response to neoadjuvant chemotherapy. These features were histogram parameters, GLCM, and GLRLM, with GLRLM accounting for the majority (Supplemental Table 2). A rad_score was established based on the above 25 radiomics features using Extra-Trees method. Age and preoperative $M$ status were found to be significantly different between responding group and non-responding group (both $P<0.05$ ) (Table 2), and thus a clinical score was built based on them. By integrating the rad_score and two clinical variables, a rad_clinical_score was derived using SVM algorithm. Results showed that the rad_score (Odds ratio [OR] $=1.21 \times 10^{5}, 95 \%$ confidence interval [Cl]: $52.3-3.07 \times 10^{9}, P<0.01$ ) was significantly associated with the treatment response of neoadjuvant chemotherapy (Table 3 ), and the rad_clinical_score was marginally associated with treatment response $(P=0.06)$, whereas the clinical score was not $(P=0.28)$.

\section{Model performance in response prediction and validation}


The rad_score was effective in predicting responders to neoadjuvant chemotherapy in the training cohort (AUC: 0.77, 95\% Cl: 0.65-0.88) and in the validation cohort (AUC: 0.82, 95\% Cl: 0.67-0.98) (Figure 3). Compared to the rad_score, the clinical score was poorer in predicting accuracy without significant difference (training: $0.70,95 \% \mathrm{Cl}: 0.58-0.82, P=0.15$; validation: $0.62,95 \% \mathrm{Cl}: 0.42-0.83, P=0.09$ ), and the rad_clinical_score did not demonstrate an improved performance (training: $0.70,95 \% \mathrm{Cl}$ : 0.58-0.82, $P=0.12$; validation: $0.70,95 \% \mathrm{Cl}: 0.51-0.88, P=0.16$ ) (Figure 3). The DCA showed that the rad_score had the higher overall net benefit compared with the rad_clinical_score and clinical score across the majority of the risk of responders (Figure 4). Other detailed predicting performance is described in Table 4.

\section{Survival stratification by the models}

In the whole cohort, univariable and multivariable Cox regression analyses showed that the rad_score (Hazard Ratio [HR] $=0.22,95 \%$ Cl: 0.11-0.42, $P<0.01$ ) was significantly associated with OS (Table 5). Univariable analysis showed that preoperative T status ( $\mathrm{HR}=2.59,95 \% \mathrm{Cl}: 1.03-6.53, P=0.04)$, the total number of dissected lymph nodes ( $\mathrm{HR}=1.03,95 \% \mathrm{Cl}: 1.00-1.06, P=0.04)$, and postoperative $\mathrm{N}$ status (HR=2.09, 95\% Cl: 1.48-3.98, $P<0.01)$ were significantly associated with OS. Based on these clinical variables, the clinical_score was updated and also found to be significantly associated with $O S(H R=2.65$, $95 \% \mathrm{Cl}$ : 1.07-6.54, $P=0.03$ ). Furthermore, the rad_clinical_score was also updated by integrating the rad_ score with the new selected clinical variables, and was found to be associated with OS (HR=2.65, 95\% Cl: 1.07-6.54, $P=0.03$ ). Based on the threshold of rad_score of 0.59 , patients were divided into groups either with high-score or with low score. The OS in patients from the high-score group was significantly higher than that in patients from the low-score group $(P<0.001)$ (Figure 5a). Similarly, the high-score groups stratified by the rad_clinical_score $(P<0.001)$ and clinical score $(P<0.001)$ both achieved longer OS than the low-score groups (Figure $5 b, 5 c$ ).

\section{Discussion}

Our study constructed and validated an effective CT-radiomics score for predicting treatment response to neoadjuvant chemotherapy in patients with potentially resectable or limited metastatic gastric cancer. The rad_clinical_score which was derived by combining clinical variables with radiomics features, could not further improve the predicting performance when compared to the rad_score. Moreover, the rad_score was capable to stratify patients into two groups with different survival outcomes.

To the best of our knowledge, this is the first attempt we develop radiomics scores to predict the response to neoadjuvant chemotherapy in patients gastric cancer before treatment. Given the great therapeutic efficacy of neoadjuvant chemotherapy for responding patients and high risk of non-response in patients [11], the early identification of potentially responding patients who might benefit from neoadjuvant chemotherapy is important to maximise treatment efficacy and optimise personalised therapy. Our established rad_score performed well in this respect, indicating the possibility of radiomics in predicting treatment response of neoadjuvant chemotherapy for gastric cancer. Several studies were conducted previously on the texture or radiomics analysis in the evaluation of treatment response in gastric cancer. 
Jiang et al. developed a radiomics signature which was effective in predicting chemotherapy efficacy in patients with stage II and III gastric cancer [30]. Yoon et al. showed that texture features on CT images were correlated with the prognosis in patients with HER2-positive advanced gastric cancer who received trastuzumab-based treatment, with heterogeneous features suggestive of better survival outcomes [31]. Therefore, the underlying reason for our good model performance might be the fact that intratumoural heterogeneity reflected by radiomic features was associated with tumour biology and even cell cycle regulating pathways, which are strong factors influencing the efficacy of neoadjuvant chemotherapy [3234]. The full mechanism behind the relationship between radiomic features and neoadjuvant chemotherapy has not been elucidated, and radiogenomics studies are warranted to provide evidence in this issue [35]. Besides, by integrating clinical variables with radiomics features, the derived rad_clinical_score could not show superior predicting performance to that of the rad_score. This indicated that radiomics features were the stronger component of this combined score while clinical data had limited impact in elevating the performance.

In addition, our rad_score was capable to stratify patients into two groups with different risks of death, which helped us identify the subgroup of patients with poor prognosis for whom more intensified treatment and closer follow-up schedule was needed. Low rad_score was associated with poor prognosis, which made sense because low rad_score was associated with no or poor response to neoadjuvant chemotherapy. It was reported that patients who responded to neoadjuvant chemotherapy had a higher likelihood to receive curative gastrectomy, and their survival was expected to be better than that of nonresponding patients [5-9]. The finding that the rad_score developed using the outcome of treatment response to neoadjuvant chemotherapy was effective in prognosis stratification, further confirmed its clinical significance and usefulness. Instead of two models, our single model could be used in both the prediction of treatment response and survival stratification.

Previous studies have found that radiomics features were closely related to tumour biology and microscopic structure [36-39]. Our study identified 25 radiomic features associated with treatment response to neoadjuvant chemotherapy for gastric cancer. These were histogram parameters, GLCM, and GLRLM with more than half of the features being GLRLM. GLCM and GLRLM were important markers of intra-tumour homogeneity, because they represented the level of signal heterogeneity in a lesion in the manner of relative relationship between the distribution and site of the gray level. These values (GLCM and GLRLM) were higher in patients with no response to neoadjuvant chemotherapy, which indicated that the intratumoral heterogeneity was more apparent in these patients than in the responding patients. Many studies have reported that tumours with greater intratumoral heterogeneity tended to be more aggressive in terms of proliferation, metastasis, and angiogenesis [22, 40], and thus might be more resistant to neoadjuvant chemotherapy.

There are several limitations in our study. First, the sample size was small considering the relatively large number of variables. Therefore, Extremely Randomised Tree method was used to minimise the bias because it used the whole training sample rather than a bootstrap replica to build a tree, and it included a random subset of features and split nodes by choosing cut-points at random within each tree. Second, 
our models lacked the external validation, which reduced the confirmation strength of the model accuracy.

\section{Conclusion}

The radiomics score developed in this study was effective in predicting treatment response to neoadjuvant chemotherapy and stratifying patients' prognosis for gastric cancer. These findings may help clinicians in identifying potentially responding patients and providing personalised treatment.

\section{Abbreviations}

\begin{tabular}{ll} 
CT & computed tomography \\
\hline MDCT & Multi-detector computed tomography \\
\hline ULN & upper limit of normal \\
\hline BSA & body surface area \\
\hline TRG & tumor regression grading \\
\hline PACS & picture archiving and communication system \\
\hline ROI & region of interest \\
\hline GLCM & grey level co-occurrence matrix \\
\hline GLRLM & grey level run-length matrix \\
\hline GLSZM & gray level Size Zone Matrix \\
\hline SVM & support vector machine \\
\hline PCA & principle component analysis \\
\hline AUC & area under the curve \\
\hline DCA & decision curve analysis \\
\hline OS & overall survival
\end{tabular}

\section{Declarations}

- Ethical approval and consent to participate: Institutional Review Board approval was obtained. And because of the retrospective nature of the study, written informed consent from patients was waived by the Institutional Review Board because.

- Consent to publish: Not applicable. 
- Availability of data and materials: Data would be available from the corresponding author on reasonable request.

- Competing interests: The authors of this manuscript declare no relationships with any companies, whose products or services may be related to the subject matter of the article.

- Funding: This work is supported by the National Natural Foundation of China (81672343 and 81871915 , Recipient: Jian-Bo Xu), the Natural Science Foundation of Guangdong Province (No. 2017A030313570, Recipient: Jian-Bo Xu), the Natural Science Foundation of Guangdong Province (No. 2018A030310326, Recipient: Kai-Yu Sun), the Natural Science Foundation of Guangdong Province (No. 2018A030310282, Recipient: Shu-Ling Chen), the Guangdong Medical Science and Technology Foundation (A2018280, Recipient: Kai-Yu Sun) and Science and Technology Program of Guangzhou (No. 201607010050, Recipient: Jian-Bo Xu). The funding source had no involvement in the design of the study and collection, analysis, and interpretation of data and in writing the manuscript.

\section{- Authors' contributions:}

KYS and HTH: Original draft and Project administration;

SLC, JNY, GHL and LDC: Data curation;

JJP, STF, YJY, XH and HW: Resources and Supervision;

$\mathrm{XL}$ and TFW: Methodology and Formal analysis;

WW and JBX: Conceptualization, Review \& editing;

All authors have read and approved the final manuscript.

- Acknowledgements: Not applicable.

- Authors' Information: 1. Department of Gastrointestinal Surgery, The First Affiliated Hospital of Sun Yatsen University, Guangzhou, China, 510080; 2. Department of Medical Ultrasonics, Institute of Diagnostic and Interventional Ultrasound, The First Affiliated Hospital of Sun Yat-sen University, Guangzhou, China, 510080; 3. Department of Radiology, The First Affiliated Hospital of Sun Yat-sen University, Guangzhou, China, 510080; 4. GE Healthcare China, Shanghai, 200000.

\section{References}

[1] Freddie B, Jacques F, Isabelle S, et al (2018) Global cancer statistics 2018: GLOBOCAN estimates of incidence and mortality worldwide for 36 cancers in 185 countries. CA Cancer J Clin 68: 394-424.

[2] Degiuli M, Sasako M, Ponti A, Calvo F (2004) Survival results of a multicentre phase II study to evaluate D2 gastrectomy for gastric cancer. Br J Cancer 90:1727-1732. 
[3] Sano T, Sasako M, Yamamoto S, et al (2004) Cancer surgery: morbidity and mortality results from a prospective randomized controlled trial comparing $\mathrm{d} 2$ and extended para-aortic lymphadenectomyJapan Clinical Oncology Group study 9501. J Clin Oncol 22:2767-2773.

[4] Takahashi T, Saikawa Y, Kitagawa Y. Gastric cancer: current status of diagnosis and treatment (2013) Cancers 5:48-63.

[5] Cunningham D, Allum WH, Stenning SP, et al (2006) Perioperative chemotherapy versus surgery alone for resectable gastroesophageal cancer. N Engl J Med 355:11-20.

[6] Ychou M, Boige V, Pignon JP, et al (2011) Perioperative chemotherapy compared with surgery alone for resectable gastroesophageal adenocarcinoma: an FNCLCC and FFCD multicenter phase III trial. J Clin Oncol 29:1715-1721.

[7] Glimelius B, Ekstrom K, Hoffman K, et al (1997) Randomized comparison between chemotherapy plus best supportive care with best supportive care in advanced gastric cancer. Ann Oncol 8:163-168.

[8] Aoyama T, Nishikawa K, Fujitani K, et al (2017) Early results of a randomized two-by-two factorial phase II trial comparing neoadjuvant chemotherapy with two and four courses of cisplatin/S-1and docetaxel/cisplatom/S-1 as neoadjuvant chemotherapy for locally advanced gastric cancer. Ann Oncol 28: 1876-1881.

[9] Xue K, Ying XJ, Bu ZD, et al (2018) Oxaliplatin plus S-1 or capecitabine as neoadjuvant or adjuvant chemotherapy for locally advanced gastric cancer with D2 lymphadenectomy: 5-year follow-up results of a phase II-III randomized trial. Chin J Cancer Res 30: 516-525.

[10] Al-Batran SE, Homann N, Pauligk C, et al (2017) Effect of neoadjuvant chemotherapy followed by surgical resection on survival in patients with limited metastatic gastric or gastroesophageal junction cancer: the AIO-FLOT3 trial. JAMA Oncol 3: 1237-1244.

[11] Xiong BH, Cheng Y, Ma L, Shang CQ (2014) An updated meta-analysis of randomized controlled trial assessing the effect of preoperative chemotherapy in advanced gastric Cancer. Cancer Invest 32:272284.

[12] Weber WA, Ott K, Becker K, et al (2001) Prediction of response to preoperative chemotherapy in adenocarcinomas of the esophagogastric junction by metabolic imaging. $\mathrm{J}$ Clin Oncol 19: 3058-3065.

[13] Wieder HA, Ott K, Lordick F, et al (2007) Prediction of tumor response by FDG-PET: comparison of the accuracy of single and sequential studies in patients with adenocarcinomas of the esophagogastric junction. Eur J Nucl Med Mol Imaging 34: 1925-1932.

[14] Hansen ML, Fallentin E, Lauridsen C, et al (2014) Computed tomography (CT) perfusion as an early predictive marker for treatment response to neoadjuvant chemotherapy in gastroesophageal junction cancer and gastric cancer-a prospective study. PloS One 9: e97605. 
[15] Lee SM, Kim SH, Lee JM, et al (2009) Usefulness of CT volumetry for primary gastric lesions in predicting pathologic response to neoadjuvant chemotherapy in advanced gastric cancer. Abdom Imaging 34:430-440.

[16] Ang J, Hu L, Huang PT, et al (2012) Contrast-enhanced ultrasonography assessment of gastric cancer response to neoadjuvant chemotherapy. World J Gastroenterol 18:7026-7032.

[17] Giganti F, De Cobelli F, Canevari C, et al (2014) Response to chemotherapy in gastric adenocarcinoma with diffusion-weighted MRI and (18) F-FDG-PET/CT: correlation of apparent diffusion coefficient and partial volume corrected standardized uptake value with histological tumor regression grade. J Magn Reson Imaging 40:1147-57.

[18] Schneider PM, Eshmuminov D, Rordorf T, et al (2018) ${ }^{18}$ FDG-PET-CT identifies histopathological nonresponders after neoadjuvant chemotherapy in locally advanced gastric and cardia cancer: cohort study. BMC Cancer 18: 548.

[19] Kumar V, Gu Y, Basu S, et al (2012) Radiomics: the process and the challenges. Magn Reson Imaging 30:1234-1248.

[20] Li Y, Liu X, Xu K, et al (2018) MRI features can predict EGFR expression in lower grade gliomas: A voxel-based radiomic analysis. Eur Radiol 28:356-362.

[21] Esteva A, Kuprel B, Novoa RA, et al (2017) Dermatologist-level classification of skin cancer with deep neural networks. Nature 542:115-118.

[22] Braman NM, Etesami M, Prasanna P, et al (2017) Intratumoral and peritumoral radiomics for the pretreatment prediction of pathological complete response to neoadjuvant chemotherapy based on breast DCE-MRI. Breast Cancer Res 19:57.

[23] Yang L, Dong D, Fang MJ, et al (2018) Can CT-based radiomics signature predict KRAS/NRAS/BRAF mutations in colorectal cancer? Eur Radiol 28:2058-2067.

[24] Mandard AM, Dalibard F, Mandard JC, et al (1994) Pathologic assessment of tumor regression after preoperative chemoradiotherapy of esophageal carcinoma. Clinicopathologic correlations. Cancer 73: $2680-2686$.

[25] Noble F, Lloyd MA, Turkington R, et al (2017) Multicentre cohort study to define and validate pathological assessment of response to neoadjuvant therapy in oesophagogastric adenocarcinoma. $\mathrm{Br} \mathrm{J}$ Surg 104: 1816-1828.

[26] Qiao X, Jiao H (2018) Data mining techniques in analyzing process data: a didactic. Front Psychol 9: 2231. 
[27] Laster L (1967) Statistical background of methods of principle component analysis. J Periodontol 38: Suppl 649-666.

[28] Geurts P, Ernst D, Wehenkel L (2006) Extremely randomized trees. Machine Learn 63: 3-42.

[29] Maree R, Geurts P, Wehenkel L (2007) Random subwindows and extremely randomized trees for image classification in cell biology. BMC Cell Biol 8 Suppl 1: S2.

[30] Jiang YM, Chen CL, Xie JJ, et al (2018) Radiomics signature of computed tomography imaging for prediction of survival and chemotheapeutic benefits in gastric cancer. EbioMedicine 36: 171-182.

[31] Yoon SH, Kim YH, Lee YJ, et al (2016) Tumor heterogeneity in human epidermal growth factor receptor 2 (HER2)-positive advanced gastric cancer assessed by CT texture analysis: association with survival after trastuzumab treatment. Plos One 11: e0161278.

[32] Aerts HJ, Velazquez ER, Leijenaar RT, et al (2014) Decoding tumour phenotype by noninvasive imaging using a quantitative radiomics approach. Nat Commun 5:4006.

[33] Tan P, Yeoh KG (2015) Genetics and Molecular Pathogenesis of Gastric Adenocarcinoma. Gastroenterology 149: e3.

[34] O'Connor JP, Aboagye EO, Adams JE, et al (2017) Imaging biomarker roadmap for cancer studies. Nat Rev Clin Oncol 14:169-186.

[35] Mazurowski MA (2015) Radiogenomics: what it is and why it is important. J Am College Radiol 12: 862-866.

[36] Grossmann P, Stringfield O, El-Hachem N, et al (2017) Defining the biological basis of radiomic phenotypes in lung cancer. Elife 6: e23421.

[37] Fox MJ, Gibbs P, Pickles MD (2016) Minkowski functionals: An MRI texture analysis tool for determination of the aggressiveness of breast cancer. J Magn Reson Imaging 43:903-910.

[38] Ganeshan B, Goh V, Mandeville HC, Ng QS, Hoskin PJ, Miles KA (2013) Non-small cell lung cancer: histopathologic correlates for texture parameters at CT. Radiology 266:326-336.

[39] Segal E, Sirlin CB, Ooi C, et al (2007) Decoding global gene expression programs in liver cancer by noninvasive imaging. Nat Biotechnol 25:675-680.

[40] Wang WT, Yang L, Yang ZX, et al (2018) Assessment of microvascular invasion of hepatocellular carcinoma with diffusion kurtosis imaging. Radiology 286: 571-580

\section{Tables}

Table 1. Clinicopathological characteristics of the training and validation cohorts 


\begin{tabular}{|c|c|c|c|}
\hline Factors & Training cohort $(n=74)$ & Validation cohort $(n=32)$ & $P$ value \\
\hline Age (years, mean \pm SD) & $55.15 \pm 11.43$ & $54.13 \pm 12.68$ & 0.68 \\
\hline Gender & & & 0.53 \\
\hline Male & 48 & 18 & \\
\hline Female & 26 & 14 & \\
\hline BMI $\left(\mathrm{kg} / \mathrm{m}^{2}\right)$ & $21 \pm 3$ & $22 \pm 3$ & 0.12 \\
\hline Preoperative T stage & & & 0.39 \\
\hline 2 & 1 & 0 & \\
\hline 3 & 11 & 2 & \\
\hline 4 & 62 & 29 & \\
\hline Preoperative N status & & & 0.99 \\
\hline 0 & 2 & 1 & \\
\hline $1-3$ & 72 & 31 & \\
\hline Preoperative M status & & & 0.85 \\
\hline 0 & 47 & 19 & \\
\hline 1 & 27 & 13 & \\
\hline Postoperative T stage & & & 0.83 \\
\hline $1-2$ & 11 & 6 & \\
\hline $3-4$ & 63 & 26 & \\
\hline Postoperative N status & & & 0.06 \\
\hline 0 & 35 & 8 & \\
\hline $1-3$ & 39 & 24 & \\
\hline Postoperative M status & & & 0.90 \\
\hline 0 & 53 & 24 & \\
\hline 1 & 21 & 8 & \\
\hline Postoperative TNM stage & & & 0.23 \\
\hline 0 & 3 & 3 & \\
\hline 1 & 6 & 1 & \\
\hline 2 & 22 & 5 & \\
\hline 3 & 22 & 15 & \\
\hline 4 & 21 & 8 & \\
\hline $\operatorname{AFP}(\mathrm{ng} / \mathrm{mL})$ & $9.25 \pm 17.79$ & $6.48 \pm 8.33$ & 0.40 \\
\hline CEA (IU/L) & & & 0.23 \\
\hline Normal & 50 & 26 & \\
\hline Elevated & 24 & 6 & \\
\hline CA125 (IU/L) & & & 0.08 \\
\hline Normal & 73 & 29 & \\
\hline Elevated & 1 & 3 & \\
\hline CA199 (IU/L) & & & 1.00 \\
\hline Normal & 64 & 27 & \\
\hline Elevated & 10 & 5 & \\
\hline Operative duration (min) & $349.46 \pm 116.47$ & $359.22 \pm 111.98$ & 0.69 \\
\hline Blood transfusion (ml) & $347.92 \pm 506.39$ & $362.50 \pm 458.43$ & 0.89 \\
\hline \multirow{2}{*}{ Total number of dissected lymph node } & $40.81 \pm 18.67$ & $44.09 \pm 17.77$ & 0.40 \\
\hline & Page $15 / 21$ & & \\
\hline
\end{tabular}




\begin{tabular}{llll}
\hline Number of positive lymph node & $5.27 \pm 9.01$ & $7.34 \pm 10.42$ & 0.30 \\
\hline Treatment response & & & 0.25 \\
\hline TRG 1 & 3 & 3 & \\
\hline TRG 2 & 34 & 14 & \\
\hline TRG 3 & 24 & 10 & \\
\hline TRG 4 & 10 & 4 & \\
\hline TRG 5 & 3 & 1 & \\
\hline
\end{tabular}

Abbreviations: $\mathrm{BMI}=$ body mass index; $\mathrm{PS}=$ performance status; $\mathrm{AFP}=$ alpha-fetoprotein; $\mathrm{CEA}=$ carcinoembryonic antigen; TRG=tumor regression grading.

Table 2. Comparison of clinical variables and radiomics score in the responding group and non-responding group in the training cohort

\begin{tabular}{|c|c|c|c|}
\hline Irs & Responding group & Non-responding group & $P$ value \\
\hline rears, mean \pm SD) & $56.76 \pm 11.42$ & $52.85 \pm 11.91$ & 0.02 \\
\hline er & & & 1.00 \\
\hline ə & 24 & 24 & \\
\hline ale & 13 & 13 & \\
\hline $\left.\mathrm{kg} / \mathrm{m}^{2}\right)$ & $21 \pm 3$ & $21 \pm 4$ & 0.42 \\
\hline $\mathrm{ng} / \mathrm{mL})$ & $8.95 \pm 13.51$ & $7.86 \pm 17.55$ & 0.56 \\
\hline$(\mathrm{IU} / \mathrm{L})$ & & & 0.80 \\
\hline nal & 26 & 24 & \\
\hline ated & 11 & 13 & \\
\hline 9 (IU/L) & & & 1.00 \\
\hline nal & 32 & 32 & \\
\hline ated & 5 & 5 & \\
\hline $5(\mathrm{IU} / \mathrm{L})$ & & & 1.00 \\
\hline nal & 36 & 37 & \\
\hline ated & 1 & 0 & \\
\hline serative $\mathrm{T}$ stage & & & 0.17 \\
\hline & 0 & 1 & \\
\hline & 8 & 3 & \\
\hline & 29 & 33 & \\
\hline serative $\mathrm{N}$ status & & & 0.19 \\
\hline & 2 & 0 & \\
\hline & 13 & 8 & \\
\hline & 18 & 21 & \\
\hline & 4 & 8 & \\
\hline serative $\mathrm{M}$ status & & & 0.05 \\
\hline & 28 & 19 & \\
\hline & 9 & 18 & \\
\hline Imics score & $0.54 \pm 0.22$ & $0.41 \pm 0.22$ & $<0.01$ \\
\hline
\end{tabular}


Abbreviations: $\quad \mathrm{BMI}=$ body mass index; $\mathrm{PS}=$ performance status; $\mathrm{AFP}=$ alpha-fetoprotein; $\mathrm{CEA}=$ carcinoembryonic antigen.

Table 3. Association of the three scores with treatment response of neoadjuvant chemotherapy for gastric cancer

\begin{tabular}{|c|c|c|c|c|c|}
\hline$\overline{\mathrm{rts}}$ & Models & $\begin{array}{l}\text { Responding } \\
\text { group }\end{array}$ & $\begin{array}{l}\text { Non-responding } \\
\text { group }\end{array}$ & OR $(95 \% \mathrm{CI})$ & $P$ value \\
\hline \multirow[t]{3}{*}{ ing cohort } & rad_score & $0.56 \pm 0.26$ & $0.38 \pm 0.25$ & $14.51(2.40,98.35)$ & $<0.01$ \\
\hline & clinical_score & $0.56 \pm 0.11$ & $0.47 \pm 0.13$ & $355.62\left(7.98,2.41 * 10^{4}\right)$ & $<0.01$ \\
\hline & $\begin{array}{l}\text { rad_clinical } \\
\text { score }\end{array}$ & $-0.61 \pm 0.29$ & $-0.88 \pm 0.34$ & $12.22(2.79,64.65)$ & $<0.01$ \\
\hline \multirow{4}{*}{$\begin{array}{l}\text { ation } \\
\mathrm{t}\end{array}$} & rad_score & $0.54 \pm 0.12$ & $0.42 \pm 0.08$ & $1.21 * 10^{5}$ & $<0.01$ \\
\hline & & & & $\left.3.07 * 10^{9}\right)$ & \\
\hline & clinical_score & $0.52 \pm 0.12$ & $0.48 \pm 0.11$ & $33.46\left(0.07,2.98 * 10^{4}\right)$ & 0.28 \\
\hline & $\begin{array}{l}\text { rad_clinical } \\
\text { score }\end{array}$ & $-0.38 \pm 0.23$ & $-0.56 \pm 0.27$ & $16.90(1.04,422.82)$ & 0.06 \\
\hline
\end{tabular}

Abbreviations: $\mathrm{OR}=$ odds ratio, $\mathrm{CI}=$ confidence interval.

Table 4. Predictive performance of the three scores in the treatment response of neoadjuvant chemotherapy for gastric cancer in the validation cohort

\begin{tabular}{lllllllllllll}
\hline & Cut-off & ACC & & SEN & & SPE & & PPV & \multicolumn{3}{c}{ NPV } \\
\cline { 2 - 13 } & - & ACC & $P$ & SEN & $P$ & SPE & $P$ & PPV & $P$ & NPV & $P$ \\
\hline rad_score & 0.516 & 0.81 & - & 0.75 & - & 0.88 & - & 0.86 & - & 0.78 & - \\
clinical_score & 0.462 & 0.63 & 0.11 & 0.75 & 1.00 & 0.50 & $<0.01$ & 0.60 & 0.02 & 0.67 & 0.32 \\
\hline rad_clinical score & -0.651 & 0.69 & 0.26 & 0.88 & 0.18 & 0.50 & $<0.01$ & 0.64 & 0.04 & 0.80 & 0.84 \\
\hline
\end{tabular}

Abbreviations:

ACC=accuracy; SEN =sensitivity; $\mathrm{SPE}=$ specificity; PPV=positive predictive value; NPV=negative

predictive value.

Table 5. Multivariable analysis of the three scores and clinicopathological characteristics with overall survival 


\begin{tabular}{llll}
\hline Factors & HR & 95\% CI & $P$ value \\
\hline Preoperative T stage & 2.59 & $1.03-6.53$ & 0.04 \\
Total number of dissected lymph node & 1.03 & $1.00-1.06$ & 0.04 \\
\hline Postoperative N status & 2.09 & $1.48-3.98$ & $<0.01$ \\
\hline TNM stage & 2.67 & $1.15-6.23$ & 0.02 \\
\hline rad_score & 0.22 & $0.11-0.42$ & $<0.01$ \\
\hline clinical_score & 2.65 & $1.07-6.54$ & 0.03 \\
\hline rad_clinical_score & 4.27 & $1.18-15.39$ & 0.03 \\
\hline
\end{tabular}

Abbreviations: $\mathrm{HR}=$ hazard ratio; $\mathrm{CI}=$ confidence interval.

\section{Figures}



\section{Figure 1}

Flow diagram of study population. 



Figure 2

A female patient was diagnosed as gastric cancer (T4aN2M0). CT before neoadjuvant chemotherapy (A) showed a mass-type tumor measured $25 \mathrm{~mm}$ in maximal depth and $80 \mathrm{~mm}$ in maximal length. CT after neoadjuvant chemotherapy (B) showed a shrunken mass measured $14 \mathrm{~mm}$ in depth and $40 \mathrm{~mm}$ in length. Pathology examination after surgery (C) showed the ROI delineated manually on figure A (D) showed residual tumor tissue (arrow) and infiltrated inflammatory cells (arrow head).

Training cohort

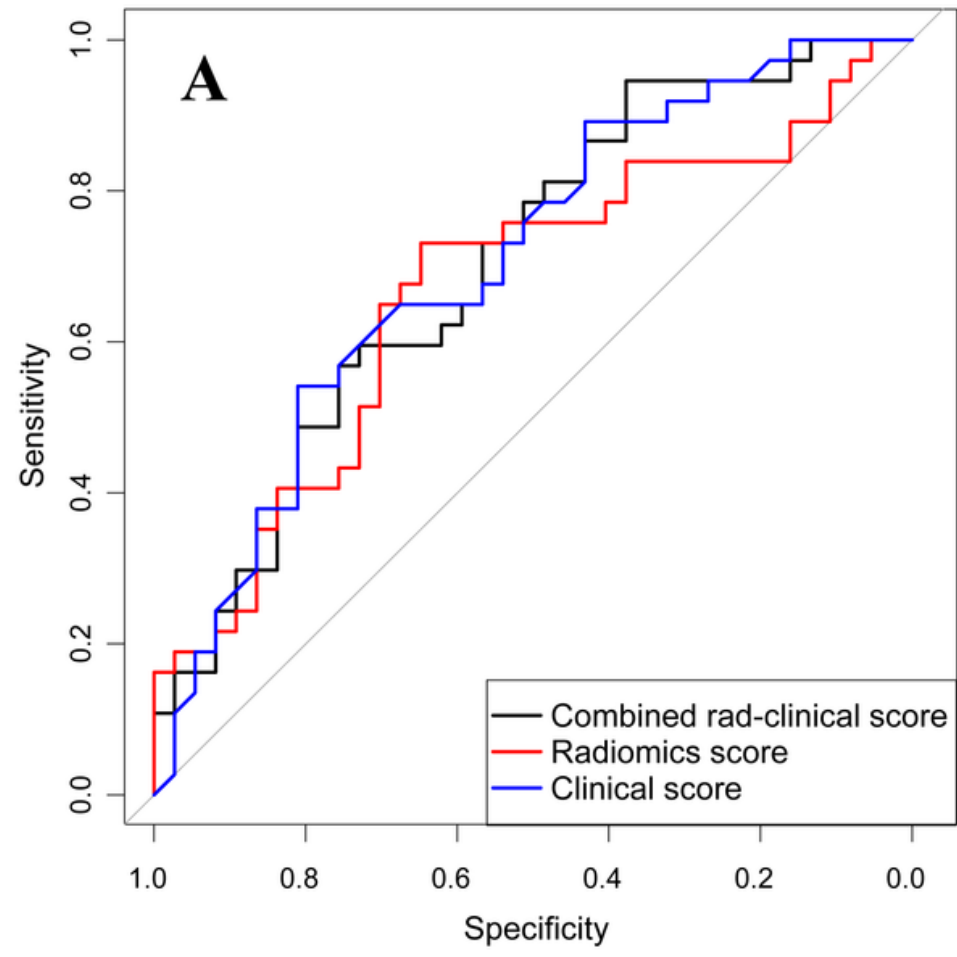

Validation cohort

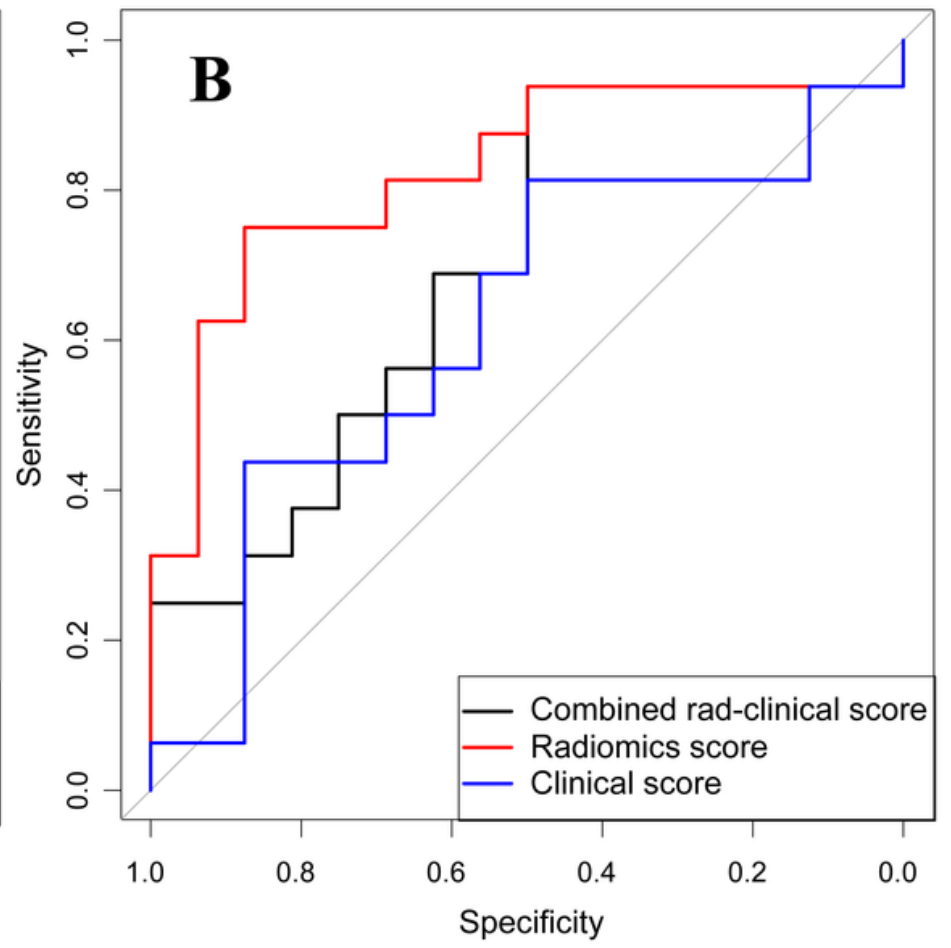

Figure 3

Receiver operating characteristics curves of the three scores in the training and validation cohorts. (a) in the training cohort; (b) in the validation cohort. 


\section{Decision Curve Analysis}

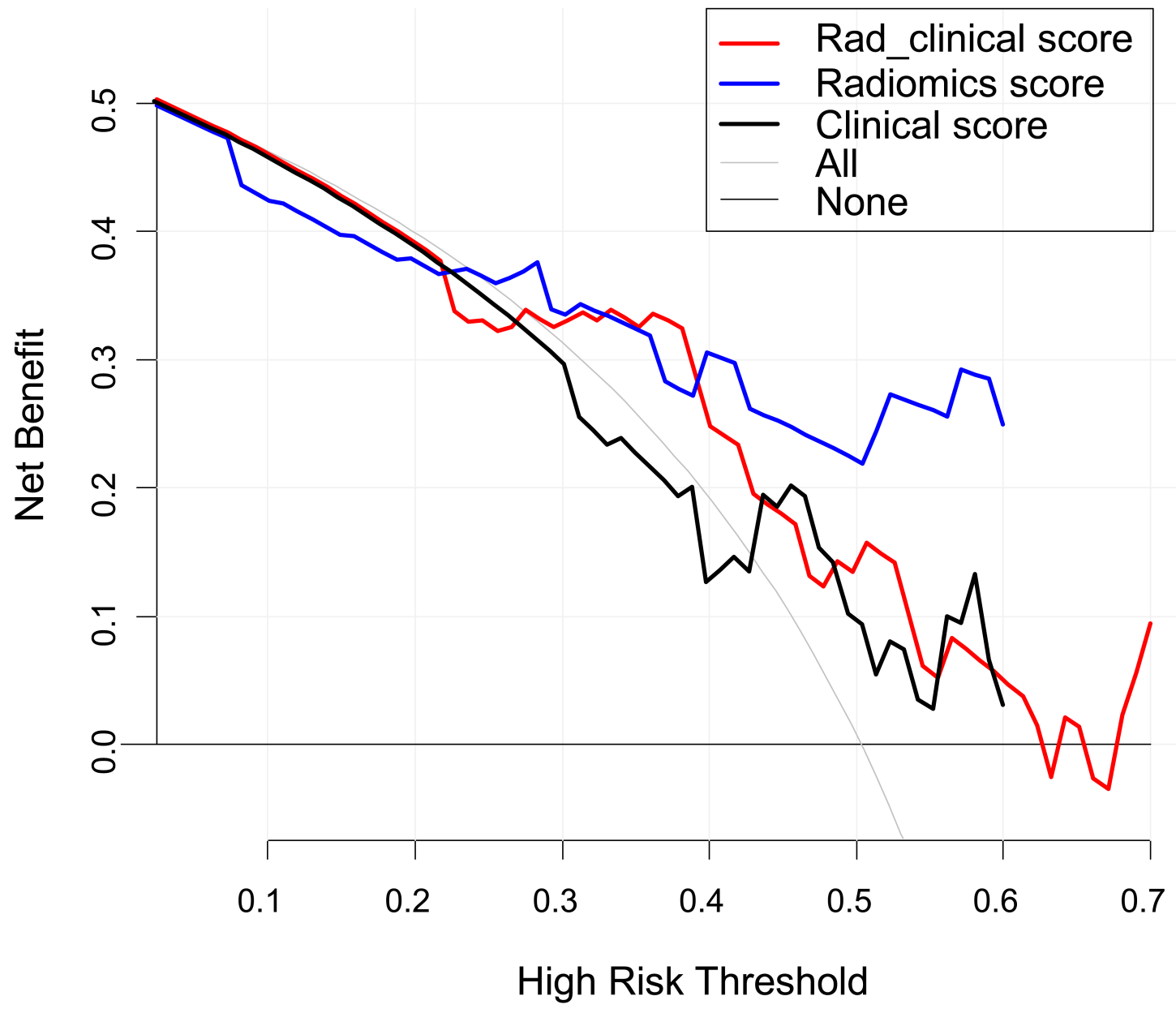

Figure 4

Decision curve analysis for the rad_score, clinical score and rad_clinical score.
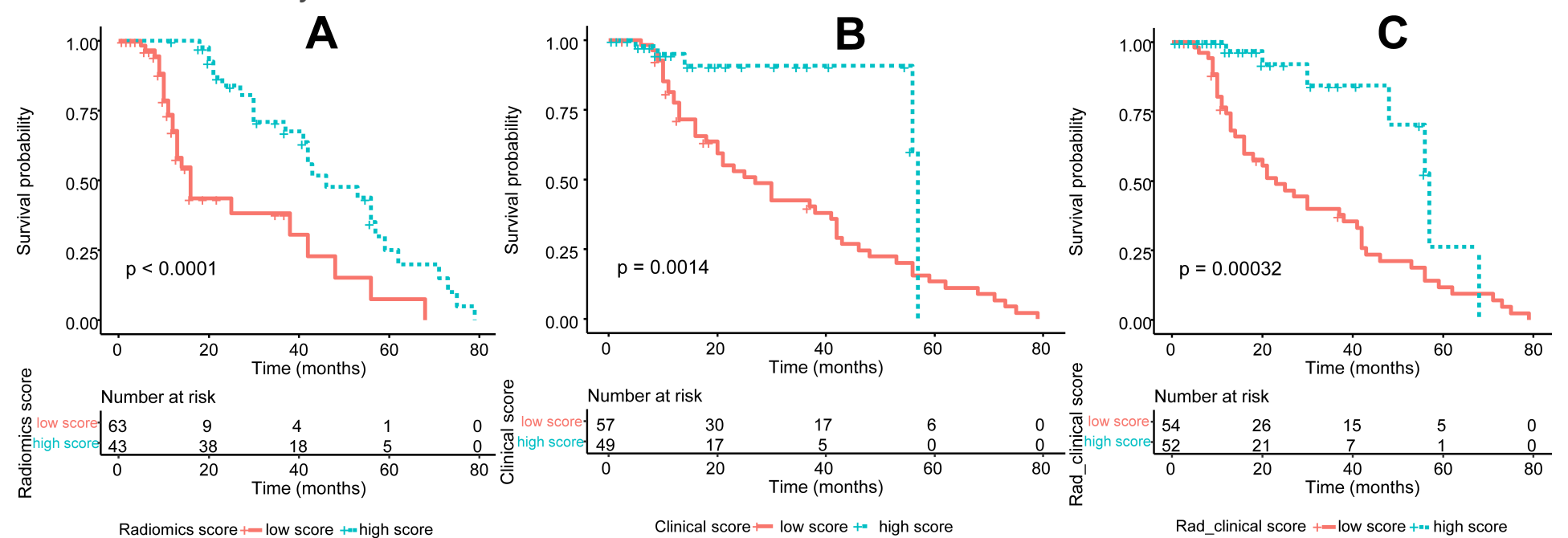

Clinical score - low score $+=$ high score

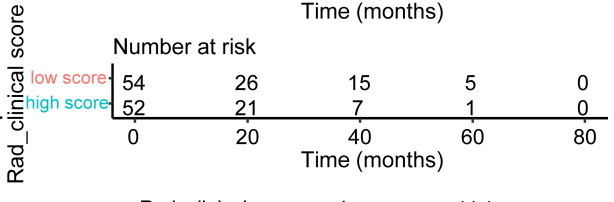

Figure 5 
Comparisons of the overall survivals between high-score group and low-score group respectively stratified by rad_score, clinical score and rad_clinical score. (a) stratified by rad_score; (b) stratified by clinical score; (c) stratified by rad_clinical_score.

\section{Supplementary Files}

This is a list of supplementary files associated with this preprint. Click to download.

- 4SupplementalTables.docx 